\title{
Topographic comparative study of paranasal sinuses in adult horses by computed tomography, sinuscopy, and sectional anatomy
}

\author{
D. De Zani • S. Borgonovo • M. Biggi • S. Vignati • \\ M. Scandella $\cdot$ S. Lazzaretti $\cdot$ S. Modina $\cdot$ D. Zani
}

Published online: 12 May 2010

(C) Springer Science+Business Media B.V. 2010

\begin{abstract}
Clinical and radiographic investigations of paranasal sinuses in horses are difficult due to the complex anatomy of these regions, the lack of patognomonic symptoms, and the low sensitivity of conventional diagnostic techniques. The aim of this study was to produce an anatomical atlas to support computed tomography (CT) and sinuscopy of the paranasal sinuses of the adult horse. Transverse, sagittal, and dorsal CT images were acquired, and sinuscopy with both rigid and flexible endoscopes was performed. The heads were frozen and sectioned using a band saw, with the cuts aligned as close as possible with the CT transverse slices. Each CT image was compared with its corresponding anatomical section and sinuscopy image to assist in the accurate identification of specific structures.
\end{abstract}

Keywords Horse $\cdot$ Paranasal sinuses $\cdot$ Computed tomography $\cdot$ Sinuscopy

\author{
Abbreviations \\ CT computed tomography \\ MRI magnetic resonance imaging
}

\section{Introduction}

Diseases of the paranasal sinuses are rare in horses, but they are of special interest in equine practices due to the difficulties of diagnosis and treatment (Freeman et al. 1990). These difficulties are correlated to the lack of specific clinical signs and to the low sensitivity of diagnostic tools, such as radiographic examination and sinucentesis (Freeman et al. 1990). Second-level diagnostic techniques achieve more detailed information about the noxa

D. De Zani $(\bowtie) \cdot S$. Borgonovo $\cdot$ M. Biggi $\cdot$ D. Zani

Dip. di Sc. Cl. Vet., Sez. di Rad. Vet. Cl. e Sper, Milano, Italy

e-mail: donatella.dezani@unimi.it

S. Vignati $\cdot$ M. Scandella $\cdot$ S. Lazzaretti

Dip. di Sc. Cl. Vet., Sez. di Cl. Chir Vet, Milano, Italy

S. Modina

Dip. di Sc.Animali, Sez di. Anatomia ed Istologia Vet. Univ. degli Studi di Milano, Milano, Italy 
pathogena and involved structures. Even the use of computed tomography (CT) and magnetic resonance imaging (MRI) is currently limited in equine practice. These imaging techniques provide excellent details of both bony and soft tissues structures. The major advantage of CT and MRI as compared with conventional radiographs is the lack of superimposition due to the ability to obtain images on sagittal, dorsal, and transverse planes directly or through multiplanar reconstructions. Endoscopic examination with a rigid or flexible endoscope is a useful diagnostic tool that permits direct evaluation of internal structures (Tucker and Farrell 2001; Arencibia et al. 2000). Sinus endoscopy has an additional value for use as a therapeutic technique (formalin intralesional injection, sinus lavages, drainages, etc.). Few reports about the tomographic anatomy of paranasal sinuses have been published (Morrow et al. 2000; Solano and Brawer 2004). In addition, a multimodal approach is desirable to obtain earlier and more accurate diagnoses. The aim of this study was to give the equine practitioner a comparative tool that allows the correlation between CT images, sinuscopy, and anatomic features.

\section{Materials and methods}

CT scans (PQ2000S, Philips, Italy) on dorsal, sagittal, and transverse planes of three adult (8-11 years old) equine heads was performed. Scan settings were: Thick /index; $5 \mathrm{~mm}, \mathrm{~mA}$; $175, \mathrm{kV} ; 120 / 130$; scan time: $1 \mathrm{sec}$ with a bone algorithm. Following the scanning procedures, a sinus endoscopy with both rigid (5.5 mm endoscope; Stortz; St. Louis, MO) and flexible (pediatric video bronchoscope; Fujinon; Wayne, NJ) endoscopes was performed. Afterwards, the heads were frozen at $-20^{\circ} \mathrm{C}$ and sliced by band saw along the same transverse plane used during CT scan. Each slice was cleaned and photographed.

\section{Results}

A total of 9 anatomic slices, $44 \mathrm{CT}$ transverse images, $46 \mathrm{CT}$ sagittal images, $33 \mathrm{CT}$ dorsal images, and 21 videoendocope frames were obtained and afforded a comparative atlas of the paranasal sinus region. The atlas was divided into two sections organized in tables. In the first section, each CT transverse image was compared with its corresponding anatomic sections (Fig. 1, left) and relevant structures were identified and labeled. In the same section, schematic drawings for endoscope orientation and corresponding endocope images (Fig. 1, right) were included. The second section of the atlas consisted of tomographic images on dorsal (Fig. 2, left) and sagittal planes (Fig. 2, right). Each CT image was completed by an image with a reference line for the scan plane.

\section{Discussion}

The compilation of different imaging techniques and gross sectional anatomy allowed for a better understanding of the normal anatomy of the paranasal sinus region. The relationship between these complex structures was easily visualized. Excellent anatomical details, including good discrimination between bony structures and soft tissues, were evident in the CT images (Arencibia et al. 2000). The main advantage of CT in comparison with survey radiography is the lack of superimposition of anatomical structures. CT images were acquired on transversal, sagittal, and dorsal planes. No previous studies have reported 

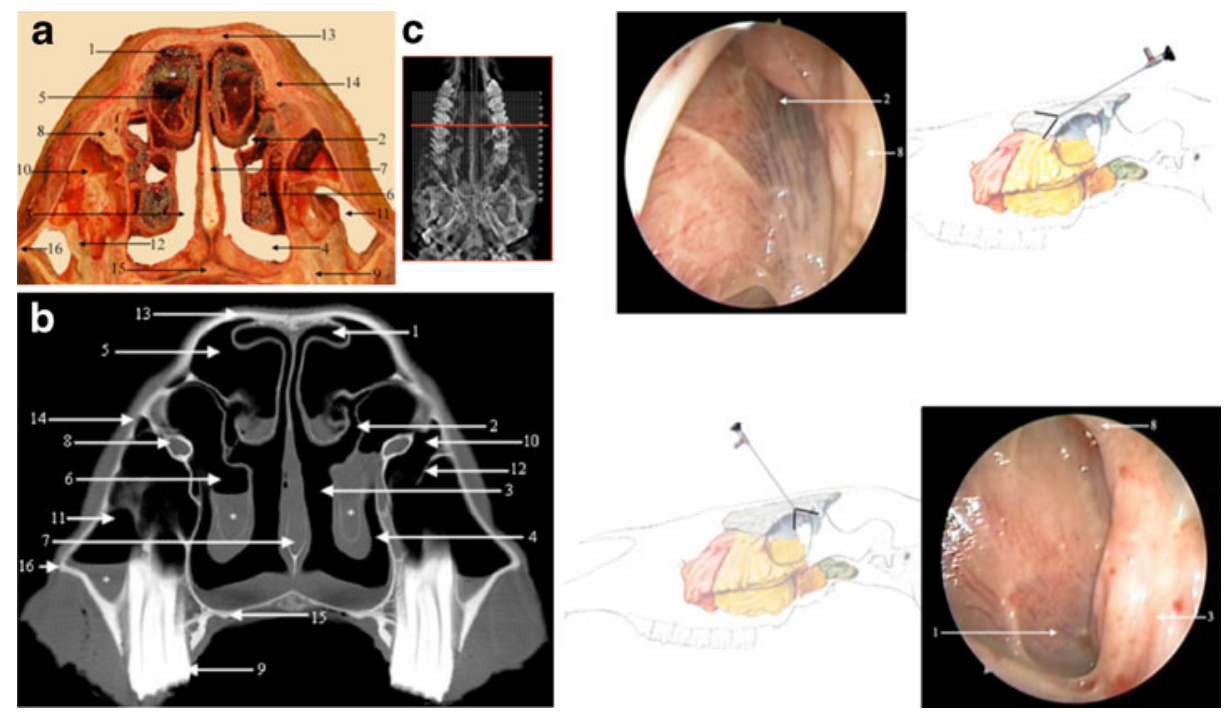

Fig. 1 (Left panel) anatomic section (a) and corresponding tomographic image (b). Scan and sectional planes are displayed in a dorsal reference image (c, red line). (Right panel) sinuscopic view from frontal sinus portal with schematic drawing for endoscope orientation: 1) Caudal portion of frontal sinus; 2) Rostral portion of frontal sinus; 3) ethmoid; and 8) Ventral bulla

positionings different from the one used for transverse image acquisition (Morrow et al. 2000). Sagittal images could be obtained only on cadaver heads or through post-processing reconstruction. Dorsal image acquisition could be performed by flexing the head and tilting the CT gantry. The positioning described could also be used "in-vivo." Endoscopic examination allowed a detailed visualization of equine sinuses, providing a useful diagnostic and a therapeutic tool.

Both rigid and flexible endoscopes were used. The advantages of using a rigid arthroscope, described by McIlwraith (2005) and Ruggles et al. (1991), are the low
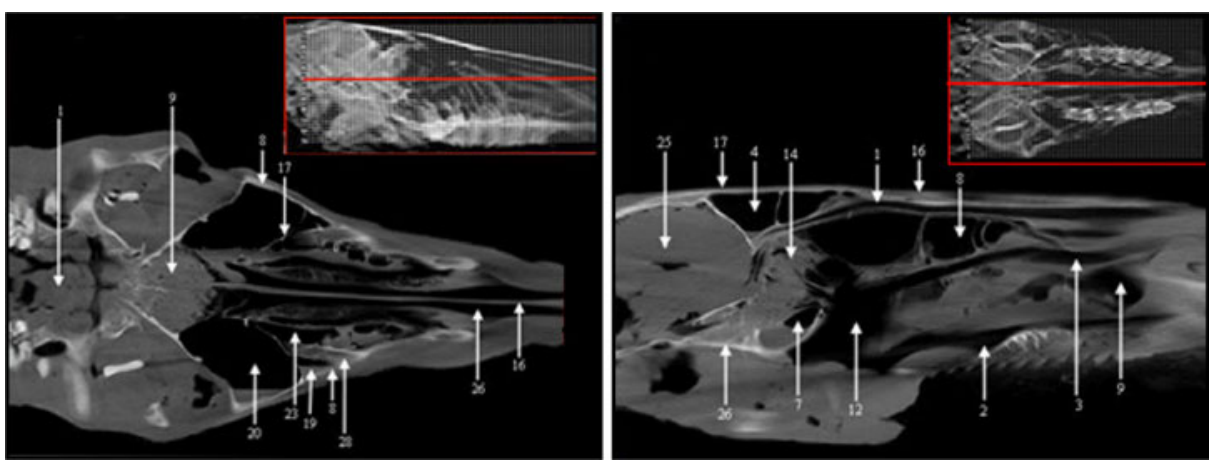

Fig. 2 (Left panel): CT images on dorsal plane: 1) Cerebrum; 8) Maxillary bone; 9) Ethmoid; 16) Nasal septum; 17) Septum of the maxillary sinus; 19) Rostral maxillary sinus; 20) Caudal maxillary sinus; 23) Ventral nasal concha; 26) Common nasal meatus; 28) Infraorbital canal. (Right panel): CT image on median sagittal plane: 1) Dorsal nasal meatus; 2) Ventral nasal meatus; 3) Middle nasal meatus; 4) Conchofrontal sinus; 7) Sphenopalatine sinus; 8) Dorsal nasal concha; 9) Ventral nasal concha; 12) Nasopharynx; 14) Ethmoid; 16) Nasal bone; 17) Frontal bone; 25) Cerebrum; 26) Pre-sphenoid 
purchase and maintenance costs. The main disadvantage of this technique is the limited range of motion of the instrument that does not allow the investigation of the entire sphenopalatine sinus. With a flexible endoscope, an investigation of the complete paranasal sinus region is possible, but this instrument is limited due to high costs. The work presented in this paper will be a useful support diagnostic tool for interpretation of tomographic images for the equine practitioner. The multimodal approach offers a valid interpretation of the paranasal sinus region. Because of the anatomical differences between adult and foals, further studies are necessary to describe normal changes related to age in young horses. Scintigraphic examination could be useful and complementary to CT in association with a fusion imagine technique for investigation of sinus diseases particularly of dental origin (Barakzai et al. 2006).

\section{References}

Arencibia A, Vasquez JM, Rivero M, Latorre R, Sandoval JA, Vilar JM, Ramirez JA (2000) Computed tomography of normal cranioencephalic structures in two horses. Anatomia, Histologia, Embryologia 29:295-299

Barakzai S, Tremaine H, Dixon P (2006) Use of scintigraphy for diagnosis of equine paranasal sinus disorders. Vet Surgery 35:94-101

Freeman DE, Orsini PG, Ross MW, Madison JB (1990) A large frontonasal bone flap for sinus surgery in the horse. Vet Surgery 19:122-130

McIlwraith C (2005) Other uses for arthroscopy in the horse. In: Diagnostic and Surgical Arthroscopy in the Horse, 3rd edn. pp. 221-222

Morrow KL,Park RD, Spurgeon TL, Stashak TS, Arceneaux B (2000) Computed tomographic imaging of the equine head. Vet Radiol Ultrasound 41:491-497

Ruggles A, Ross MW, Freeman DE (1991) Endoscopic examination of normal paranasal sinuses in horses. Vet Surgery 20:418-423

Solano M, Brawer RS (2004) CT of the equine head: technical considerations, anatomical guide, and selected diseases. Clin Tech Equine Pract 3:374-388

Tucker LT, Farrell E (2001) Computed tomography and magnetic resonance imaging of the equine head. Vet Clinics of N Am 17:131-144 